\title{
LOS TEJIDOS RETICULARES: ALIANZAS Y SOLIDARIDADES EMPRESARIALES EN LA TERRITORIALIZACIÓN DEL MODELO DE agronegocios (Córdoba, Argentina)
}

City, island rurality and public policies. Interconnected networks: alliances and business solidarity in the territorialization of the agribusiness model

(Córdoba, Argentina)

\section{Sofía Ambrogi \\ sofi.kest@gmail.com}

Secretaría de Ciencia y Técnica / Centro de Investigaciones María Saleme de Burnichón-Universidad Nacional de Córdoba

RESUMEN: Las discusiones en torno a las transformaciones en el agro, producto de innovaciones biotecnológicas, el aumento del precio de los commodities a nivel internacional y las reformas políticas y económicas en diferentes países latinoamericanos, ha sido ampliamente trabajado en estos últimos años desde diversos campos disciplinares. Dada esta preponderancia de las transformaciones rurales en Argentina como objeto de estudio en la academia, este monográfico convoca a repensar la ruralidad como objeto de reflexión desde las ciencias sociales, prestando particular atención a las especificidades que asumen estos cambios en prácticas y experiencias rurales concretas. En ese sentido, este trabajo se pretende sumar a las discusiones que plantean tensiones en las visiones dicotómicas respecto a lo rural y lo urbano como territorios diferenciados y diferenciables. Dado que el modelo de agronegocios plantea configuraciones reticulares entre acciones estatales, intervenciones desde las empresas y asociaciones técnicas del agro, y las comunidades locales urbanas, es necesario prestar atención a los proyectos y acciones solidarias desarrollados por actores empresariales del agro, desde los cuales apuntan a construir narrativas sobre la ruralidad, el desarrollo productivo, la sustentabilidad y la responsabilidad social. Se apunta a entender cómo se construye legitimación social del modelo de agronegocios en centros urbanos y ciudades de Córdoba, Argentina, desde las intervenciones empresariales de una asociación técnica del agro. 
Palabras clave: Modelo de agronegocios; redes solidarias; territorialización; hegemonía; prácticas empresariales.

ABSTRACT: Discussions around transformations in agriculture, the result of biotechnological innovations, increased world commodity prices, and the political and economic reforms in Latin American countries, has been studied widely in recent years from diverse disciplinary perspectives. Given the prevalence of rural transformations in Argentina in the literature, this dossier calls for a rethink of rurality as an object of reflection from the social sciences, with particular attention to the specificities assumed by these changes in certain rural practices and experiences. This article aims to contribute to the discussions that raise tensions in the dichotomous view of the rural and the urban as differentiated and differentiable territories. The agribusiness model proposes network configurations between state actions, interventions from companies and agricultural technical associations, and urban local communities. As a result, attention must be given to projects and actions of solidarity undertaken by agricultural business actors, designed to construct narratives about rurality, productive development, sustainability and social responsibility. The study aims to understand how the social legitimization of the agribusiness model is constructed in the urban centers and cities of Córdoba, Argentina, through the lens of the business interventions of a technical agricultural association.

KeYWORDs: Agribusiness model, solidarity networks, territorialization, hegemony, business practices. 


\section{Introducción}

$\mathrm{D}$ ebido a las transformaciones en la estructura agraria en América Latina durante las últimas décadas, la ruralidad se ha vuelto un vasto campo de estudio dentro de las Ciencias Sociales, en particular desde la antropología y sociología rural. Pensar la ruralidad en términos de espacio únicamente circunscrito al campo y los ambientes agroproductivos, muchas veces obtura poder comprender el agronegocio como modelo que funciona reticularmente, incorporando una multiplicidad de actores y de escalas que se conjugan asimétricamente, creando territorios donde lo rural y lo urbano se amalgaman.

La intención de este trabajo es comenzar a entender este tejido reticular a partir del análisis de prácticas empresariales en ciudades de la provincia de Córdoba, Argentina, provincia del corazón pampeano atravesado por distintos procesos de agriculturalización desde mitades del siglo xx en adelante. Dado que el modelo de agronegocios (MA en adelante) plantea configuraciones reticulares entre acciones estatales, intervenciones desde las empresas y asociaciones técnicas del agro, y las comunidades locales urbanas, es necesario prestar atención a los proyectos y acciones solidarias desarrollados por actores empresariales del agro; desde ellos, apuntan a construir narrativas sobre la ruralidad, el desarrollo productivo, la sustentabilidad y la responsabilidad social. Se trata de entender cómo se construye la legitimación social del MA en centros urbanos y ciudades de Córdoba, desde las intervenciones empresariales de una asociación técnica del agro.

Se comenzará por una descripción acotada de las transformaciones de la estructura agraria en Argentina durante las últimas décadas, haciendo una recuperación crítica de un marco conceptual particular que ha enmarcado varios estudios realizados desde las ciencias sociales: el neoxtractivismo. Una revisión crítica de este marco conceptual será útil para atender a los debates que tensionan la mirada sobre las ciudades como territorios "marginales" a la hora de pensar la ruralidad y las transformaciones territoriales planteadas por el MA.

Para poder plantear una mirada crítica acerca de la dicotomía ruralidad-urbanidad, se describirá brevemente la conformación de las llamadas "ciudades sojeras" o agro-ciudades de la región pampeana, con el fin de comprender por 
qué su crecimiento se encuentra atravesada por las transformaciones del MA, y la razón por la cual sectores empresariales ligados al agro (agroindustrias, agroalimentarias y asociaciones técnicas) desarrollan en ellos diversos proyectos, programas y acciones solidarias.

Realizar un análisis sobre estas prácticas empresariales puede ayudar a la hora de entender las complejas configuraciones mediante las cuales el mundo empresarial crea la legitimidad social del MA. Se considera necesario comprender de qué modo se producen y reproducen prácticas y discursos acerca de la ruralidad -ligada ésta a una cultura laboral-y una visión innovadora del desarrollo productivo; cómo se entiende el rol de las empresas del agro en la formación de ciudadanos y prácticas responsables y sustentables; y complejizar el rol del estado y las acciones estatales en la formulación de normativas que apuntan a garantizar y desarrollar intervenciones diversas entre empresas, asociaciones y las comunidades locales.

Pensando este amplio abanico de actores y experiencias, se hace indispensable volver al nudo gordiano de la dicotomía rural-urbano desde enfoques antropológicos que proponen el estudio de prácticas empresariales, ya que en ellas se evidencian los anclajes locales de prácticas y discursos a diferentes escalas. Se abordarán estas prácticas empresariales desde proyectos y programas desarrollados por dos empresas: la multinacional agroindustria Monsanto, y el grupo empresarios y técnicos de Asociación Argentina de Consorcios Regionales de Experimentación Agrícola (AACREA) en una ciudad de la provincia de Córdoba.

Este trabajo se enmarca dentro en una investigación doctoral, así como también comparte reflexiones elaboradas desde el trabajo de un equipo de investigación. Se recuperan observaciones y registros de trabajo de campo, análisis documental y entrevistas desarrolladas durante el período 2014-2017.

\section{Agronegocios y Neoextractivismo: disputas y legitimaciones en los territorios en transformación}

Las transformaciones en la matriz productiva de los países latinoamericanos, producto de políticas neoliberales y que generaron profundos cambios 
en la estructura agraria, han sido explicadas por diversos intelectuales como producto de una dinámica particular: la "acumulación por desposesión" (Harvey, 2004). Esta dinámica explica un proceso de reordenamiento espacial y productivo, generando, entre otras consecuencias, una ampliación de la frontera agraria, la transformación de economías locales en enclaves productivos a través de la privatización de tierras y recursos colectivos, la supresión de formas no capitalistas de producción y la expulsión de campesinos, chacareros y otros actores sociales de los territorios rurales.

El modelo que esta dinámica encarna y sustenta, ha sido conceptualizado como neoextractivista por diversos autores (Svampa, 2013; Gudynas, 2009, entre otros). Se considera una variante nueva del extractivismo clásico (propio de la minería y la extracción de hidrocarburos), que se caracteriza por la explotación de grandes volúmenes de recursos naturales exportados como commodities, y dependen de economías de enclave, localizadas o espacialmente extendidas.

Lejos de representar renovados intereses por el desarrollo de las producciones locales, el extractivismo exportador responde a las demandas del mercado internacional bajo una lógica de reprimarización de la economía. El neoextractivismo supone la existencia de grandes empresas transnacionales que dominan sectores claves de la producción y comercialización, así como las tecnologías punta utilizadas en estos procesos. Si bien constituye un modelo remitido originalmente a la extracción de minerales -potenciado en años recientes por la minería a cielo abierto- también tiene que ver con la extracción del petróleo y con cierto tipo de agricultura como el modelo sojero y otros identificados como agronegocio. El desarrollo neoextractivista es generador de ventajas comparativas que se visibilizan en el crecimiento económico de pocos actores, e incrementa asimetrías y conflictos sociales, económicos y ambientales.

Si bien muchos de estos elementos ayudan a caracterizar las transformaciones agrarias, hay algunos de sus puntos que obstaculizan interpretar cambios en territorios concretos. Diversos autores cuestionan la reprimarización de la economía a partir de este tipo de producciones, ya que no se tienen en cuenta las dinámicas de redistribución del excedente agrario ni la interpelación dinámica entre territorios rurales y urbanos. Pensar el agrone- 
gocio como dinámica neoextractivista no permite abarcar qué sucede efectivamente con la población excedente y sobrante (¿Hacia dónde van? ¿Qué hacen? ¿Cómo reconvierten sus actividades productivas?), ni con las regalías generadas a través de la venta y exportación de los commodities (¿El capital se fuga? ¿Cómo se traducen éstas en políticas públicas?). Al centrar la mirada únicamente sobre lo que sucede en el campo se pierde de vista qué pasa paralelamente en las "periferias", es decir, los territorios urbanos, pues se piensa en la trama productiva únicamente vinculada a las materias primas (Gago, 2014; Gago y Medrazza, 2015).

Haciendo hincapié en el rol del Estado - que desde muchas perspectivas se considera como compensador o neointervencionista-, estas lecturas invitan a pensar en la venta de commodities y la circulación interna de sus derrames (gracias a políticas sociales producidas desde sus excedentes) como diferentes caras de la misma moneda. Ambas construyen modos particulares de vida en los territorios. Poder centrar el ojo analítico sobre otros procesos y esferas relacionadas con el consumo y con la circulación de riqueza, implica entonces también una mirada crítica sobre una tendencia que, por una parte, suele victimizar a los "perdedores" del nuevo paradigma (campesinos, chacareros, productores familiares) y, por otra, a tratar las poblaciones urbanas como pasivas y meras receptoras de políticas públicas y planes sociales financiados desde el agronegocio. Es así como también se plantea una fuerte crítica a categorías como la "acumulación por desposesión", ya que se opaca por un lado la categoría misma de explotación y, por otro, se desconoce la producción de valor de esas poblaciones que las propias finanzas ya evalúan como no marginales. El marco explicativo del extractivismo suele poner demasiado énfasis en los conflictos y tensiones sociales, más que en los consensos y legitimaciones que produce el MA.

Estudiar espacios y procesos de las últimas décadas vinculados a la ruralidad, implica necesariamente reconocer una serie de transformaciones que dieron lugar al MA, al que las autoras Gras y Hernández (2013, 2016a, 2016b) han caracterizado minuciosamente en diversas instancias. Desde la década de 1970 en adelante existieron cambios en los procesos productivos de la estructura agraria (en términos de subordinación de la producción agraria a la industria, expresados en avances tecnológicos y biotecnológicos). Respecto a 
lo que interesa abordar en este momento, es imprescindible entender las innovaciones en los procesos de gestión (nuevas tecnologías de la comunicación e información, profesionalización de la administración, organización de la empresa en red, etc.) y de nuevas identidades profesionales, y por lo tanto institucionales del agro (Gras y Hernández, 2013). Rescatando puntualmente lo que respecta a los pilares organizacionales e ideológicos, resulta necesario hacer hincapié sobre la modalidad del trabajo en "red" de los nuevos actores dominantes del agro (en mayor o menor medida), ya que las alianzas a nivel transectorial generaron convergencias materiales y simbólicas expresadas en nuevas identidades, discursos y prácticas (Gras y Hernández, 2016). Las transformaciones en el mapa institucional del agro tienen así un rol fundamental, porque expresan cambios en las modalidades de organización colectiva y de la construcción identitaria de los actores agropecuarios (Gras y Hernández, 2016; Liaudat, 2016), que a su vez tiene influencia sobre las maneras de presentarse y vincularse intra y extra sectorialmente.

Respecto a la modalidad de "red", hay que subrayar que si bien

(...) durante el primer decenio de esta transformación la red se consolida como espacio de intercambio entre actores económicos (tercerizadores de servicios, proveedores de insumos, financiadores, clientes, etc.) del sector, en un segundo momento, que abarca la última década, la red se extiende más allá de las fronteras del sector, tejiendo alianzas con distintos actores sociales y conformando flujos de conocimiento y prácticas hacia los territorios en los que éstos se insertan. (Córdoba, 2015, p. 34)

Esta incorporación torna relevante comenzar a indagar acerca de las formas específicas que asume la relación entre las empresas del sector y el resto de la sociedad civil, ya que es allí donde se manifiestan importantes elementos que hacen de "la construcción de la relación" no solamente la aspiración hegemónica del grupo, sino el modelo mismo. Hay que pensar el MA no como actor, sino como lógica específica de producción y acumulación, que redefine tanto el control de la tierra, como el capital, el trabajo o la organización de la producción, mediante anclajes locales, regionales y nacionales.

Lo que se intenta recuperar en este artículo es, por lo tanto, una mirada amplia sobre territorios y poblaciones urbanas como parte de la dinámica del "agro" (no meramente subsidiarios), así como la necesidad de pensar 
este modelo desde su tejido reticular intra y extra-sectorialmente. Interesa particularmente pensar el agro como territorio fuertemente interpretado por el mundo urbano, así como también se piensa la ruralidad desde las urbes (en formato de agronegocio, como "faro de innovación productiva", o como “cuna de buenas prácticas agropecuarias", entre otras concepciones).

\section{La territorialización del modelo de agronegocios: una ruralidad polisémica}

Para seguir las líneas de los debates desarrollados anteriormente, interesa considerar cómo el agronegocio ancla relaciones y redes en las ciudades, desde las cuales se piensa y se (re)construye lo rural desde las escuelas, desde los centros vecinales, desde las asociaciones civiles, y desde los representantes del gobierno local; cómo en estos espacios también se construye lo rural. Poder ver cuáles son los mecanismos y dispositivos concretos de territorialización de las empresas para crear imágenes, discursos y prácticas sobre lo rural: una ruralidad ligada a la innovación productiva a la fuente de trabajo y a la sustentabilidad.

Como se ha referenciado anteriormente, muchos debates en las ciencias sociales se enfocan en el vaciamiento del "campo", ya que el campesinado, los chacareros y los productores a pequeña escala que no contaron con la posibilidad (es decir, con el know-how y el capital) de reconvertir su actividad, tuvieron que abandonar sus propiedades o espacios de vivienda.

Ese supuesto vaciamiento, que muchas veces es traducido a la imagen del "desierto verde" de la soja, lleva a pensar que existen territorios e incluso perfiles socio-productivos que efectivamente se disuelven, desaparecen. ¿Qué sucede entonces con esos sujetos? Además, las ganancias que comienza a extraerse del agro, subordinadas a una lógica transnacional condicionada por el mercado internacional, parecerían también desvanecerse a horizontes lejanos. ¿El capital efectivamente se fuga?

Algunos autores (Cloquell, 2007; Gras, 2013) constatan que a mayor producción e ingresos globales generados por la agricultura de exportación (sobre todo en oleaginosas y granos), mayor es el impulso del crecimiento y 
desarrollo económico local y regional. Esto lleva a la creación de una figura muy nombrada en círculos académicos y extra-académicos: las "ciudades sojeras", término que hace referencia a pequeños pueblos o ciudades de pocos habitantes que, gracias a la actividad agropecuaria, fueron extendiéndose en tamaño y en términos de calidad de vida, ubicadas sobre todo en las regiones pampeanas del país (Centro-Este: Santa Fe, Buenos Aires, Córdoba, La Pampa y Entre Ríos). Estas mejoras se refieren principalmente al aumento en la presencia de servicios (luz, gas, cloacas, centros de salud, cajeros automáticos y bancos, institutos de formación y escuelas, entre otros), y negocios ligados a la actividad agropecuaria, como por ejemplo los centros de acopio, las oficinas bancarias, los comercios de venta de insumos, los talleres de reparación de maquinaria,... Estos centros urbanos, que de alguna manera ya habían comenzado a tener un impulso de crecimiento bajo la primera modernización agraria desde mediados del siglo $\mathrm{xx}$, se conforman como centros de servicio de la producción agropecuaria.

En estos centros urbanos en crecimiento se fueron integrando tejidos sociales complejos, compuestos tanto por aquellos productores que migraron del campo porque decidieron hacer del alquiler un ingreso, como también aquellos que tuvieron que reconvertirse en vendedores de maquinaria, prestadores de servicios agrícolas, o simplemente comerciantes, micro-emprendedores o cuentapropistas. En este sentido, la relación entre el desarrollo agropecuario y los territorios se ha complejizado, dando lugar a la conformación de lo rural como espacio polisémico (Gras, 2013), ya que se desdibujan y entrelazan espacios urbanos y rurales, en la medida en que tradicionales sujetos del campo se instalan en ciudades o centros urbanos intermedios, y actores sin raigambres rurales comienzan a invertir e interesarse en estos territorios.

Las características de la ocupación y el empleo en estos territorios (tanto las "ciudades sojeras" como aquellas que se han dedicado históricamente a otras matrices productivas pero que comienzan a ser atravesadas por el MA) redefine los contornos entre lo rural y lo urbano. Se abre entonces un abanico de interrogantes que complejiza el estudio de la ruralidad, la cual puede entenderse como una mayor heterogeneidad, producto de nuevos cortes en la estructura social, como rasgo característico del modelo (Gras, 2013). 
Entender la composición de estas ciudades y la razón de su crecimiento (en términos poblacionales, pero también de ingresos) en las últimas décadas, nos llevan a tener una imagen más definida de las redes que desenvuelve el MA, generando nuevos territorios, re-territorializando y des-territorializando lo rural y lo urbano (Domínguez, Lapegna y Sabatino, 2006).

Esta composición de los centros urbanos pampeanos, invitan a comprender prácticas empresariales de sectores ligados al agro que, mediante proyectos, programas y acciones solidarias de diversos tipos, establecen vínculos con las comunidades locales, con quienes muchas veces no tienen relaciones de emplazamiento o laborales directas. Dichas prácticas se encuentran íntimamente entrelazadas con diversos actores que habitan y circundan la ciudad: representantes de los gobiernos locales, referentes religiosos, clubes deportivos, partidos políticos, asociaciones civiles sin fines de lucro o comunidades educativas, entre otras.

Los discursos y materialidades que circulan en estas prácticas se convierten en propiedad de los distintos "beneficiarios", aunque, claro está, desde posiciones asimétricas debido al lugar dominante que ocupan tanto en la red del MA como en el territorio. La anticipación hipotética es que estas prácticas empresariales en red, que tienen lugar en espacios "tranqueras afuera", como pueden ser las comunidades educativas urbanas, tienen que entenderse como dispositivo fundamental desde las cuales: a. los excedentes del agro se transforman en políticas públicas, y b. desde diversos formatos (acciones solidarias, proyectos educativos, intercambios regionales, actividades de formación en oficio, de líderes y emprendedores) se construyen activamente nociones acerca de la ruralidad, el mundo y la cultura productiva, y la sustentabilidad y responsabilidad social como metas a alcanzar entre actores mancomunados.

\section{Empresas y comunidades educativas: escuelas como faro de la "innovación"}

Villa del Rosario es una ciudad de la provincia de Córdoba, ubicada como cabeza departamental de Río Segundo, a 78 kilómetros al sudoeste de la 
capital provincial. Es una ciudad de aproximadamente 15.000 habitantes, y mientras que en el ejido urbano las actividades económicas principales son el comercio y la prestación de servicios, su agro se encuentra inserto en la región pampeana, siendo los cultivos principales los de soja, maíz, maní y, en menor medida, forraje destinado a la actividad ganadera. La actividad industrial ha ido creciendo desde la segunda mitad de la década pasada, concentrándose casi exclusivamente en la elaboración y creación de valor agregado de productos agropecuarios.

El avance de las fronteras agropecuarias y del monocultivo de flex-crops, generaron varios efectos. Uno de los más tangibles que se puede observar es la desaparición de biodiversidad en el caso de las abejas. Este caso fue comentado por el director de una escuela agrotécnica pública de gestión privada de la ciudad, desde la cual se venía trabajando con una sección didáctico-productiva en apicultura, y que tuvo que ser cerrada hace cuatro años por la ausencia de abejas en la zona afectada por el uso de agrotóxicos.

Debido a que esta ciudad se encuentra atravesada por la actividad agroproductiva, se observa una presencia marcada de lazos entre empresas agroindustriales, asociaciones técnicas del sector, y espacios educativos. En el marco de programas de vinculación con la comunidad y de proyectos de Responsabilidad Social Empresarial (RSE), esta escuela agrotécnica ha sido beneficiaria de distintos fondos y talleres de capacitación, tanto por la multinacional Monsanto, como por AACREA, que apadrinó a la escuela dentro de su grupo regional.

A continuación se expondrán los resultados tanto de observaciones, como de registros de campo, entrevistas y análisis de documentos que se fueron construyendo para distintos períodos (2015-2017) en esta escuela, principalmente a partir de las interacciones mantenidas con quien llamaremos Miguel, actual director de la institución.

En un principio, en los años 70, el colegio agrotécnico fue creado apuntando no tan sólo a capacitar a los hijos de productores agrícolas, sino también como espacio de contención para que los jóvenes de las zonas aledañas no migraran a las ciudades en búsqueda de trabajo. Como era el único colegio secundario de la zona, construyó un internado de varones, para poder alojar a estudiantes de diversos lugares (Capital, Montecristo, Río Primero, Río 
Segundo, Pilar, Matorrales, Costa Sacate, Las Varillas, Arroyito, Temples, Cañada Río Pinto, Carlos Paz, entre otros). La institución cuenta hoy con un internado que alberga entre 150-170 jóvenes varones de distintas regiones de la provincia de Córdoba, provenientes de familias de distintos orígenes socioeconómicos, y que se vinculan laboralmente de distintas maneras con actividades productivas agropecuarias.

Para Miguel, director de este colegio agrotécnico del corazón productivo agrario en el centro de Córdoba, la vinculación entre escuela y empresas resulta de valor fundamental para el "autosustento". Antes de ser director, coordinó desde el 2006 en adelante proyectos de inversión para el colegio, en donde buscó el "beneficio para el colegio con la participación de instituciones de la comunidad" (comunicación personal). Es así como esta escuela comenzó a involucrarse con organizaciones ligadas al agro, como Monsanto y AACREA, y otras asociaciones civiles, que permitieran mejorar las secciones didáctico-productivas presentes en el predio escolar. Los lazos entretejidos con estas empresas y asociaciones civiles y técnicas se generan desde un objetivo común, al que él caracteriza de 'transacciones 'ganar-ganar' o 'sumar-sumar", que le darían un plus al colegio, que de otra manera "no se podrían garantizar".

En efecto, los niveles tecnológicos alcanzados actualmente en las secciones didáctico-productivas se deben a la inversión por parte de empresas como Monsanto, y a las recomendaciones y el capital social brindado por AACREA, ya que el colegio cobra una cuota considera baja, y muchos chicos se encuentran becados; razones por las cuales la institución no se encuentra con el capital para invertir en las mismas. Las secciones didáctico-productivas se extienden a lo largo de cuatro hectáreas donadas por una orden religiosa Lasallana, y consisten en: huerta orgánica, vivero, monte frutal, apicultura, granja (que tiene varias partes: avicultura -gallinas, pollitos parrilleros, ñandúes- y conejos), ganadería (porcina, vacuna, bovina, caprina, ovina y yunta de llamas), industria agrícola (laboratorio de extracción y acondicionador de semen), mantenimiento, panificadora y tambo. El criterio de autosustento anteriormente mencionado, apunta a que los productos desarrollados en estas secciones satisfagan las necesidades alimentarias de los estudiantes; 
simultáneamente, muchos de sus derivados son vendidos a las familias, la población de la ciudad, y a productores de la región.

Al referirse a la incorporación de su escuela dentro del programa analizado, Miguel siempre menciona los enormes aportes que implica una modalidad de encuentro y discusión que se pone en juego en relación a los proyectos, ya que considera que, sumado a las críticas y soluciones aportadas para mejorar las secciones, los chicos se desenvuelven con grupos de personas que se encuentran insertas en el sistema productivo; con líderes, que pueden servirles de ejemplo a aquellos que piensan desenvolverse a futuro en estos sectores;.... Desde esta perspectiva los encuentros brindan una red de acceso a un capital social que quizás de otra forma no se alcanzaría, por lo menos no desde el colegio. Si bien este director reconoce que no necesariamente todos los consejos les sirven para el trabajo agrotécnico, sí han podido ir avanzando sobre ciertos proyectos que los han conectado más con productores locales de la zona, por ejemplo, en la venta de dosis de inseminación artificial porcina y vacuna.

El discurso que presenta Miguel (las palabras que enmarcan la lógica del "ganar-ganar") pareciera colarse del discurso y las lógicas de pensamiento que también se transmiten desde los intercambios con AACREA y Monsanto. Hay un relato explícito de que un país en desarrollo es resultado directo de la relación entre empresas socialmente responsables y una sociedad civil (en este caso la escuela) que se encuentra entrelazada con sectores productivos con intermediación de expertos o asociaciones técnicas (como es el caso), y que promueve un espíritu innovador y emprendedor en los jóvenes. Poder estudiar estos programas de cerca permite entender cómo se forjan alianzas estratégicas con diferentes actores sociales, de qué manera se piensan y se presentan las empresas, de qué manera piensan estas la escuela y el estado, y qué inserción plantean en relación con esta esfera.

En lo que refiere específicamente al programa EduCREA, implementado por AACREA, se destaca su desarrollo en los diversos niveles educativos, articulados no solamente desde esa institución, sino en coordinación con el sector público y asociaciones civiles, para fomentar y transferir la metodología CREA en las comunidades escolares. Se trata de trabajar no solamente en las aulas y las secciones didáctico-productivas entre miembros CREA, téc- 
nicos, maestros y estudiantes, sino también en lo que se refiere a la formación docente y de directivos escolares. Las modalidades de acción son sumamente diversas: desde la transferencia de metodología CREA en las distintas secciones didáctico-productivas, hasta la realización de foros interregionales, la elaboración de material formativo y comunicativo a través de boletines, manuales, canales de difusión por YouTube, y la realización de prácticas profesionalizantes y pasantías laborales, entre otras.

Respecto a las intervenciones de Monsanto, a través de su proyecto de RSE llamada "Semillero de Futuro", el colegio fue beneficiario de subsidios desde el 2006, subsidios que obtenían mediante la presentación de proyectos escritos por el puño de Miguel para equipar y armar las secciones didáctico-productivas. Con la colaboración de Monsanto en términos de capital, pero también de recomendaciones (a través de técnicos que establecían relaciones con directores y profesores), se abrieron secciones diversas, que apuntan a agregarle valor a las materias primas: laboratorios para acondicionamiento de semen y preparación de dosis para la venta a productores locales, sección de elaboración de panificaciones y farináceos y una sala de sección de industria alimenticias, entre otras. También es importante mencionar la realización de talleres de capacitación (para los que se proveyó del equipamiento de una Sala de Usos Múltiples) en conjunto con técnicos de la empresa e invitados especiales de ONGs relacionadas. La participación de la familia en estas actividades, que referían a la salud, la nutrición y la capacitación, generó un revuelo, dado a que estas actividades no solían realizarse en otros espacios de la ciudad. En palabras de Miguel:

Al colegio no nos cuesta por ahí pensarlo solamente en forma pedagógica, porque es un colegio que tiene un criterio de autosustento. Si vos bajas la capacidad de una sección, tenés que generar inversiones para pagar alimentos que consumen los chicos en el internado. El excedente se vende poquito, y se consume entre los docentes, los padres de los alumnos, algún ciudadano de ahí que va y compra o encarga dulces, pollos y demás. (...) Una buena impronta que le queda al alumno de generar sus propios recursos, lo digo como exalumno. El otro día voy a la sala de maestro y alumnos que venían de cursar, vendían alfajores de maicena. En mi materia le doy a los chicos en séptimo año, último del técnico, para que generen su propio microemprendimiento en las facilitaciones del cole. Esas son las cosas buenas que nos dejan las redes, las capacitaciones. No sé qué tan bachiller o técnico van a salir, pero que van a salir buscavidas, van 
a salir buscavidas. Lo importante no es sólo preocuparse de generar sus propios recursos, sino que te conduzca al cuidado del entorno, generar tus propios recursos, tratar de ser renovable en todo. (Miguel, director de agrotécnico, comunicación personal: 05/03/2016)

Junto con el cambio de estándares de políticas éticas empresariales de las últimas dos décadas (Córdoba, 2015; Giniger, 2011), los grupos CREA y Monsanto -aunque con lógicas y propuestas distintas- comenzaron a generar proyectos de compromiso de transferencia y construcción de capital social (sobre todo a partir del 2009) con comunidades educativas, jóvenes innovadores y emprendedores, científicos del sector público y privado, y representantes políticos locales. Esto puede verse plasmado en los programas EduCREA, Formación de Líderes, CREAtech, CREAlab y Grupos CREA de Intendentes (G8) en el caso de AACREA, y de los Semillero de Futuro para Monsanto, donde desarrollan proyectos de trabajo con la comunidad, invirtiendo fuertemente en financiamiento y con una lógica acentuada en la transferencia de tecnología y equipamiento de las secciones didáctico-productivas. En el desarrollo de estas iniciativas plasmadas en programas se pueden rastrear contenidos (discursivos y materiales) que hace referencia a una visión específica que tienen AACREA y Monsanto sobre la sociedad, el individuo, la tecnología, la cultura del trabajo, así como de los roles articuladores que se distribuyen entre comunidad educativa, estado y empresa. Las insistencias temáticas sobre las cuestiones agroalimentarias, las prácticas de desarrollo sustentable y el emprendedurismo, no son tampoco pensadas únicamente desde estas dos empresas, sino que responden en parte también a lineamientos de organismos internacionales que mediante convenios (principalmente el Pacto Global de Naciones Unidas -PGNU- vigente desde el año 2000) imponen estándares de medición de acciones empresariales en estos ejes. Se entiende que resulta fundamental analizar los modos en que las lógicas globales se traducen a territorios concretos: si bien el MA responde y produce bajo una lógica financiera internacional, necesariamente es al mismo tiempo producida, implementada y apropiada para contextos locales y regionales específicos, dando lugar a reconfiguraciones específicas.

Es importante mencionar cómo estas políticas de vinculación no son solamente impulsadas bilateralmente desde estos actores empresariales/ 
escuelas. En los últimos años se puede observar una mayor presencia por parte de Ministerio de Agroindustria para comenzar a intervenir en diseños de currículums escolares, presencia plasmada en la firma de convenios entre asociaciones técnicas y empresariales (como AACREA) con la Secretaría de Agricultura Familiar, Coordinación y Desarrollo Territorial. Desde esta diversidad de actores, la escuela rural y agrotécnica se posiciona como "faro" de innovación agrotécnico y productivo; y los futuros egresados, como futuros profesionales que desarrollen, articulen, innoven, produzcan y/o exporten productos agropecuarios o agrotecnológicos diversificados. La forma de llegar a esta formación de jóvenes es a través de una correcta enseñanza en "Buenas Prácticas Agropecuarias", una mirada sustentable y el desarrollo de empleo desde el emprendedurismo. En los documentos analizados se construye desde los distintos actores la imagen de las escuelas como motor de desarrollo regional, desarrollo que únicamente es posible mediante la sinergia y esfuerzos aunados de la comunidad educativa, el estado y las empresas del agronegocio.

\section{Interrogantes que se abren: perspectivas etnográficas en las prácticas empresariales}

Para comprender la instalación del MA en las comunidades es necesario explorar las dimensiones ideológicas (no únicamente productivas) que asumen las prácticas empresariales en contextos urbanos, y que se traducen a largo plazo en una aspiración hegemónica tanto dentro del sector, como también hacia la "comunidad" (es decir, sectores e instituciones no agrarias). Es importante recuperar aquí los desarrollos teóricos que incorpora la autora Soledad Córdoba (2015) quien trata de describir los modos concretos de construcción de legitimación social a partir de la creación de redes solidarias que interconectan sectores del agro argentino con otros actores e instituciones de la sociedad. El llamado "modelo en red" funciona como un dispositivo de legitimación desde el momento en que en el discurso de las megaempresas -y de los medios de comunicación y ciertos trabajos académicos- se lo muestra como equivalente al 'ganar-ganar' (o 'win-win'), previamente mencionado por el director del colegio, Miguel. Este discurso aporta a la emergencia de 
una nueva configuración ideológica que acompaña las transformaciones en las formas de acumulación capitalista hacia un formato flexible, integrando diversos actores y sectores en un mismo universo.

Los estudios sobre prácticas empresariales en el campo de las ciencias sociales se han ido multiplicando con el paso de los años: perspectivas críticas a las ciencias del management ahondan en las ambivalencias, contradicciones y potencialidades que le son inherentes a las políticas éticas y solidarias de las corporaciones. Hay que darle sentido a la emergencia de estas nuevas formas de ética capitalista corporativa, de la cual se encuentra embebida la práctica y el discurso de la RSE y las acciones solidarias. Analizar estas prácticas educativas empresariales nos permiten abordar la construcción de lenguajes comunes para hablar no solamente de la agricultura y la ruralidad en general, sino del rol empresarial en el Estado y para con la sociedad civil.

La necesidad de asumir una perspectiva etnográfica relacional, y de analizar las especificidades locales, se justifica por la sencilla razón de que no todo es posible de ser explicado por procesos globales, dado que el capital se adecua a las zonas y regiones determinadas. El MA tiene traducciones propias, y es apropiado por el conjunto de actores locales de maneras diversas: en otras palabras, tiene anclajes distintos y distintivos en los territorios. ¿Por qué entonces la insistencia en prestar atención también a actores trasnacionales, como lo puede ser el PNUD? Esto se debe a que existen procesos globales, en gran medida porque también existen actores globales, lo cual no debe analizarse desde una lógica verticalista de decantamiento de poder, sino que debería generar un afilamiento del ojo analítico frente a las redes complejas e intrincadas que el modelo de agronegocios plantea.

\section{Referencias}

Cloquell, S. (2007): Familias rurales. El fin de una historia en el inicio de una nueva agricultura, Homo Sapiens, Buenos Aires.

Córdoba, S. (2015) Viaje al corazón del negocio agrícola. Dispositivos de legitimación e intervención territorial del modelo de agronegocios en Argentina (tesis doctoral) Universidad Nacional de San Martín, Argentina. 
Domínguez, D., Lapegna, P. y Sabatino, P. (2006): «Un futuro presente: las luchas territoriales», Nómadas $\left(n^{\circ} 4\right)$, p. 239-246.

GAGo, V. (2014): La razón neoliberal: economías barrocas y pragmática popular, Tinta Limón Ediciones, Buenos Aires.

Gago, V. y Medrazza, S. (2015): «Para una crítica de las operaciones extractivas del capital. Patrón de acumulación y luchas sociales en el tiempo de la financiarización», Nueva sociedad, $\left(\mathrm{N}^{\circ} 255\right)$, p. 38-52.

Giniger, N. (2011): Así se templó el acero: estrategias de control laboral y respuestas sindicales en el emplazamiento sidero metalúrgico de Villa Constitución Implicancias dentro y fuera de la fábrica (Tesis doctoral), Universidad Nacional de Buenos Aires, Argentina.

Gras, C. (2013): «Empresarios rurales y acción política en Argentina», Estudios Sociológicos ( $\mathrm{N}^{\circ}$ 89) Vol. Xxx, p. 1-30.

Gras, C. y Hernández, V. (2013): Los pilares del Modelo Agribusiness y sus estilos empresariales, en C. Gras y V. Hernández (comps) El agro como negocio: producción, sociedad y territorios en la globalización, Buenos Aires, Biblos.

Gras, C. y Hernández V. (2016a): Radiografía del nuevo campo argentino. Del terrateniente al empresario transnacional. Buenos Aires, Siglo XXI.

Gras, C. y Hernández, V. (2016b): «Modelos de desarrollo e innovación tecnología: Una revolución conservadora», Mundo Agrario Vol. (N³6) Vol 17, p. 1-27.

Gras, C. y Sosa, A. (2013): El modelo de agronegocios de las principales megaempresas agropecuarias, en Gras, C. y Hernández, V. (comps.) El agro como negocio: Producción, sociedad y territorios en la globalización, Buenos Aires, Biblos.

Gudynas, E. (2012): «Estado compensador y nuevos extractivismos. Las ambivalencias del progresismo sudamericano», Revista Nueva Sociedad ( $\left.\mathrm{N}^{\circ} 237\right)$, p. 128-146.

Harvey, D. (2004): El nuevo imperialismo, Editorial Akal, Madrid.

LiAUDAT, D. (2015): «La construcción hegemónica de las entidades técnicas en el agro argentino: análisis de los discursos de AAPRESID y AACREA en la última década», Mundo Agrario, ( $\left.\mathrm{N}^{\circ} 32\right)$, Vol 16, p. 1-32.

Svampa, M. (2013): «Consenso de los Commodities y lenguaje de valorización en América Latina», Revista Nueva Sociedad ( $\left.\mathrm{N}^{\circ} 244\right), \mathrm{p}$. $29-46$. 